\title{
Les ouvrières des mines et des salines, entre Vosges et Jura, $\mathrm{XV}^{\mathrm{e}}-\mathrm{XVIII}{ }^{\mathrm{e}}$ siècle
}

\section{Paul Delsalle}

\section{(2) OpenEdition \\ Journals}

Édition électronique

URL : http://journals.openedition.org/abpo/426

DOI : $10.4000 / a b p o .426$

ISBN : 978-2-7535-1507-9

ISSN : 2108-6443

Éditeur

Presses universitaires de Rennes

Édition imprimée

Date de publication : 30 septembre 2007

Pagination : 67-90

ISBN : 978-2-7535-0554-4

ISSN : 0399-0826

Référence électronique

Paul Delsalle, "Les ouvrières des mines et des salines, entre Vosges et Jura, $x v^{\mathrm{e}}$-xvIII ${ }^{\mathrm{e}}$ siècle », Annales de Bretagne et des Pays de l'Ouest [En ligne], 114-3 | 2007, mis en ligne le 30 septembre 2009, consulté le 10 décembre 2020. URL : http://journals.openedition.org/abpo/426 ; DOI : https://doi.org/10.4000/ abpo.426 


\title{
Les ouvrières des mines et des salines, entre Vosges et Jura, $\mathrm{XV}^{\mathrm{e}}$-XVIII ${ }^{\mathrm{e}}$ siècle
}

\author{
Paul DeLSALLE \\ Maître de conférences en histoire moderne (HDR) \\ Université de Franche-Comté \\ laboratoire des sciences historiques EA 2273
}

Concernant les processus d'industrialisation au cours des siècles, rares sont les études portant sur la main-d'œuvre féminine en dehors des manufactures textiles avant le xVIII siècle $^{1}$. On a peu prêté attention à d'autres secteurs industriels, notamment celui des mines ou encore celui des salines, où l'on retrouve des femmes, parfois en grand nombre. Il ne s'agit aucunement de paysannes qui, de façon temporaire, louraient leurs bras mais de véritables salariées à plein temps ${ }^{2}$. Les archives des compagnies minières, de Lorraine, d'Alsace ou des Vosges, et celles, exceptionnelles, des salines de Salins dans le massif du Jura, permettent l'étude des ouvrières, dès le $\mathrm{xv}^{\mathrm{e}}$ siècle et jusqu'à la fin du XvIII ${ }^{\mathrm{e}}$ siècle. Elles soulèvent la question de la définition et du statut de l'ouvrière ou de la manœuvre, en dehors du milieu artisanal, au sein de la grande industrie ${ }^{3}$. Derrière ces deux termes assez vagues désignant la main-d'œuvre se cachent une grande variété d'emplois féminins, mais surtout des spécialisations et des responsabilités parfois très surprenantes.

1. HAUSER, Henri, Ouvriers du temps passé : XV $V^{e}$-XVle siècles, Paris, 1898, réédition Slatkine, 1982. Cf., par exemple, CASPARD, Pierre, « Les pinceleuses d'Estavayer. Stratégies patronales sur le marché du travail féminin au XvIII siècle ", Schweizerische Zeitschrift für Geschichte, vol. 36, 1986, p. 121-156.

2. Pour une brève synthèse des travaux récents, cf. DELSALLE, Paul, « Le travail des femmes à l'époque moderne ", Historiens et géographes, n 393, février 2006, p. 269-276.

3. Pour l'artisanat: DAvIs, Natalie Z., "Women in the Arts Mecaniques in sixteenthcentury Lyon ", Lyon et l'Europe. Hommes et sociétés, Lyon, Presses universitaires de Lyon, 1979, tome 1, p. 139-167; pour l'industrie du sel : WEBER, Renate, «Von « nassen und truckenen Dirnen ». Frauenarbeit in den bayerischen Salinen ", Salz macht Geschichte. Aufsätze, Herausgegeben von Manfred Treml, Wolfgang Jahn und Evamaria Brockhoff, Augsburg, Haus der Bayerischen Geschichte, p. 142-147. 


\section{Ouvrières dans les mines}

Un grand foyer industriel se situe dans le massif des Vosges, au contact de trois provinces : la Lorraine, l'Alsace et la Franche-Comté. Il s'agit de mines de métaux précieux : argent, cuivre et plomb argentifère principalement. Ces mines sont exploitées selon le système des galeries creusées, et non pas à ciel ouvert.

Aux textes, qui nous apportent des détails remettant en cause ce qui a été écrit (encore récemment) sur la vie ouvrière et minière, s'ajoutent des représentations iconographiques particulièrement exceptionnelles et précieuses puisqu'elles nous permettent de voir les ouvrières au travail. En effet, Heirich Groff, un peintre de Saint-Dié, a travaillé en 1529-1530 pour le duc de Lorraine, propriétaire de mines, dans le but de représenter fidèlement "La rouge myne de sainct Nicolas de la Croix ". Les dessins qu'il a réalisés sont conservés à l'École nationale supérieure des Beaux-Arts, à Paris, et ont été publiés à maintes reprises ${ }^{4}$. Le site minier concerné par ces dessins, celui de La Croix-aux-Mines, près de la ville vosgienne de Saint-Dié, exploite un gisement de plomb argentifère.

Parmi les commentaires de ces dessins, on peut relever les propos suivants : "L'univers de la mine d'argent de la Renaissance est très éloigné du monde de poussière, de crasse, d'accidents, de travail forcé ou de grisou, réalité de la mine de charbon, immortalisé par Émile Zola dans Germinal. " Ajoutons encore ces propos qui paraissent bien naïfs : " Nul danger particulier ne les attendait sous terre [...] les galeries étaient solides ${ }^{5}$. " Nous allons mesurer à quel point la réalité était très différente. Les archives relatives aux mines des Vosges comtoises donnent en effet une image totalement différente. Il ne faudrait pas oublier que les ouvriers travaillent dans le bruit et dans l'obscurité. L'éclairage se fait à la lampe de suif, ce qui provoque fumée et odeur forte ${ }^{6}$. Si les mineurs portent un tablier de cuir et surtout un cuir fessier c'est en raison de l'humidité des parois des galeries sur lesquelles ils s'appuient pour se reposer. Bref, la vie du mineur était probablement aussi dure au temps d'Henrich Groff qu'à celui de Zola. Les ouvriers des forges voisines sont, quant à eux, sans cesse en contact avec le charbon de bois, abondant en poussière.

Aux mines des Vosges saônoises ou comtoises, à Faucogney et à SaintBresson, l'emploi de femmes et des filles est une certitude, en 1578, mais nous n'en savons guère plus ${ }^{7}$. À Château-Lambert, où l'on recherche le

4. La mine, mode d'emploi. La myne rouge de sainct Nicolas de la Croix, [1529-1530] dessinée par Heinrich Groff, Paris, Gallimard, 1992, collection " Découvertes Albums "; commentaires d'Emmanuelle Brugerolles, Hébert Bati, Paul Benoît, Pierre Fluck, Henri Schoen.

5. La mine, mode d'emploi. La myne rouge de sainct Nicolas de la Croix... op. cit., p. 57.

6. On consomme ici trois à quatre tonnes de suif par an.

7. Arch. dép. du Doubs, B1468; cf. aussi BoHLy, Bernard, "L'exploitation minière dans les Vosges saônoises au XVI ${ }^{\mathrm{e}}$ siècle à travers les comptes ", De la mine à la forge en Franche-Comté des origines au XIX siècle, Annales littéraires de l'Université de Besançon, 1990, p. 229-257. 
cuivre $^{8}$, parmi les six à sept cents " pauvres mineurs, fondeurs et charbonniers " attestés en 1621, il y a des enfants mais aussi des femmes ${ }^{9}$. Mais que font-elles? On l'ignore presque toujours. Heureusement, quelques dossiers apportent des éléments de réponse.

En 1630, une aide financière est accordée à Barbe Valdinare ${ }^{10}$, ouvrière des mines de Château-Lambert, parce qu'elle est âgée et infirme. Sa requête est émouvante : "Remonstre humblement Barbe Valdinare qu'il y a envyron seize ans qu'elle réside au lieu de Chasteau Lambert ou elle a pendant ce temps servy aux mines et mesme auroit heu un bras rompu par une cheutte [chute] qu'elle fit en bocquant ${ }^{11}$, et comme elle est presentement reduitte à une extreme pauvreté ne pouvant plus travailler à cause de sa viellesse et debilité " elle demande une aide. On lui accorde la somme de trois gros par semaine parce qu'elle est digne de commisération ${ }^{12}$. Cette femme, Barbe Valdinare, a donc travaillé au bocard. Il s'agit d'un pilon mécanique utilisé pour concasser le minerai. On imagine aisément le bruit que cet objet provoque. Un dessin de Heinrich Groff (Figure 1) intitulé "Les pilleurs " (en fait, les pileurs) nous montre le système du bocard. On y voit une roue hydraulique qui actionne un arbre à cames pour concasser, piler, le minerai. On y remarque bien au premier plan une femme qui pousse une brouette (sur d'autres scènes, ce sont des hommes qui font cela).

Le cas de Barbe Valdinare n'est pas unique : les comptes des mines de Château-Lambert mentionnent des femmes, en particulier deux sœurs, rémunérées pour trier et laver le minerai :

"Anne et Elizabeht [sic] Lorentz ont receu deux frans six groz deux blans et demy

Figure 1

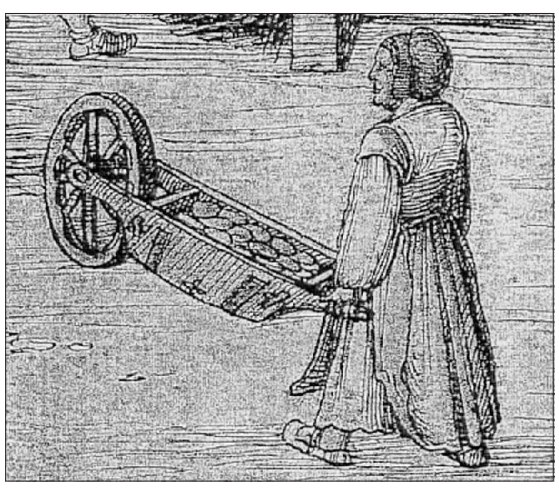

8. JEANNIN, Pierre, « Conjoncture et production de cuivre dans les Vosges méridionales à la fin du XVI et au début du XVII e siècle ", Conjoncture économique. Structures sociales. Hommage à Ernest Labrousse, Paris, 1974, p. 121-138; article repris dans Marchands d'Europe, pratiques et savoirs à l'époque moderne, Paris, Éditions Rue d'Ulm, Presses de l'École normale supérieure, 2002, p. 227-244.

9. Arch. dép. du Doubs, B 1370; sur le site minier de Château-Lambert, cf. aussi DeLSALLE, Paul, La Franche-Comté au temps des Archiducs Albert et Isabelle, 1598-1633, documents choisis et présentés, Besançon, Presses universitaires franc-comtoises, 2002, p. 259-263.

10. Notons au passage le prénom de cette femme, Barbe, qui est celui de la patronne des mineurs.

11. En travaillant au « bocard": cf. ci-dessous.

12. Arch. dép. du Doubs, B 1374. Cette mention de bocard, en 1630, est beaucoup plus ancienne que celle (1741) donnée par REY, Alain, Dictionnaire culturel en langue française, Paris, Le Robert, 2005, tome 1, p. 968. Le mot bocard a aussi un autre sens, celui de bordel (attesté en 1821) mais on ignore s'il y a un lien avec le bocard minier. 
pour vingt quatre cuviaulx et demy de bouccage qu'elles ont boucqué pour lesdittes pillattes qu'est a cinq blans le cuviaulx " (1622) ou encore : "Anne Lorentz qui garde les pillattes de jour et qui met la carnehertz [?] dessoubz les pillons a receu seyze frans trois gros pour ses gages des treyze sepmaine de ce compte qu'est a quinze gros par sepmaine ${ }^{13}$ " (1632).

Un autre dessin d'Henrich Groff représente des laveurs de minerai. Ils ont une énorme responsabilité car le lavage est l'étape ultime avant la fonte (Figure 2) :

Figure 2

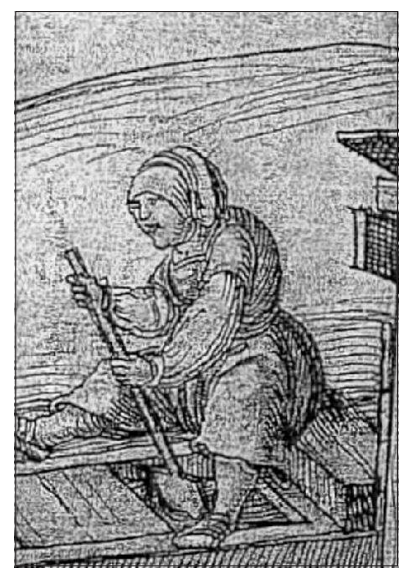

Figure 3

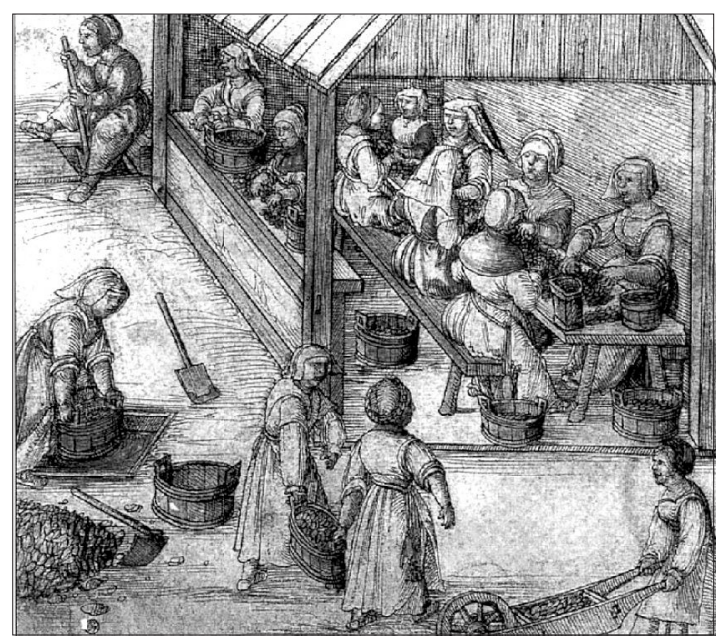

"L'opération consiste à étaler le minerai [en poudre] sur une surface de planches, faiblement inclinée, sur laquelle s'écoule de l'eau libérée par à-coups par de curieux personnages, les rameurs dont, ici, une femme. Lorsque l'eau passe sur le minerai, celui-ci est remué à l'aide d'un râble et l'eau emporte les particules légères, abandonnant le minerai pur, plus lourd ${ }^{14}$."

Sur un troisième dessin, on voit une manière de trier le minerai (Figure 3). Les femmes (ici il n'y a que des femmes) effectuent un tri purement manuel. Elles grappillent les particules de minerai pur. On les appelle cloweresses, ce qui viendrait de l'allemand klauben qui signifie littéralement égrapper. Ces deux derniers dessins montrent donc deux situations très différentes mais les femmes travaillent parmi les hommes même s'il y a des séparations parfois.

La précision quasi ethnographique des dessins montre les costumes féminins non seulement de face, mais aussi de dos ou de trois quarts. Les ouvrières sont vêtues de façon homogène mais suffisamment particularisée pour que chacune d'en-

13. Arch. dép. du Doubs, B 1380, comptes de 1622 , f ${ }^{\circ} 11$; comptes de juin-septembre $1632, \mathrm{f}^{\circ} 11$; comptes de septembre-décembre $1632, \mathrm{f}^{\circ} 2 \mathrm{v}^{\circ} ;$ ma reconnaissance s'adresse à Bernard Bohly qui m'a aimablement (en juillet 2001) indiqué cette piste archivistique.

14. La mine, mode d'emploi. La myne rouge..., op. cit. 
tre elles soit individualisée. Les vêtements se composent, semble-t-il, de deux robes tuniques superposées, qui atteignent la cheville mais ne traînent pas au sol. Sous ces robes, les femmes portent une chemise aux manches longues et bouffantes, resserrées au poignet, parfois retroussées jusqu'au coude. Seule protection visible contre le froid, une courte cape au col montant couvre les épaules et enserre le cou, ou bien un gilet sans manche, peut-être l'équivalent d'un pelisson de peau retournée, réchauffe le dos. On voit distinctement deux ceintures, faites d'une cordelette nouée à la taille, à laquelle pendent, suspendus à différents cordonnets, une bourse-aumônière et des clés. Les ouvrières sont chaussées de souliers plats, en forme de sandales et à bout rond, peut-être attachés sur le dessus par une bride.

Presque toutes cachent leurs cheveux sous une coiffe, sans doute de toile, de forme et de longueur très variées. Certains modèles ne vont pas sans rappeler ceux des servantes. En revanche, deux femmes sont tête nue, ce qui permet d'apprécier leur coiffure, de longues tresses enroulées autour du crâne. Il est difficile de décider s'il s'agit d'un signe de distinction, qui désignerait des supérieures hiérarchiques; ces ouvrières effectuent en effet des tâches identiques aux autres. La commodité fonctionnelle de ces vêtements, très semblables à ceux que portent habituellement les paysannes, est sans doute réduite. Aucun détail vestimentaire ne semble spécifiquement adapté aux opérations de tri : les femmes ne bénéficient pas de gants ni de protections de cuir, et se munissent seulement d'un léger tablier ${ }^{15}$.

Les veuves de mineurs sont secourues, dans ces mines de ChâteauLambert. Voici par exemple Etaise dont le mari, Bastien Peletier, était mineur : il est " decedez de ce monde " vers 1623. Sachant que le souverain (en l'occurrence ici l'archiduchesse Isabelle) a l'habitude « de donner quelque chose " aux femmes veuves, elle adresse une demande au trésorier des mines. Suzanne Barbie est la veuve d'un certain Claude : son mari a rendu un " bon et fidelle service " pendant seize années; elle-même a maintenant " fini sa vie", " étant vielle et ne pouvant plus gagner sa vie ", elle sollicite " quelque chose ". On lui accorde vingt sols " pour une fois ", autant dire trois fois rien. D'autres femmes reçoivent des secours lorsque leurs maris sont victimes d'un accident mortel. Barbe, veuve de Thomas Guillaume, se plaint en 1630 de n'avoir reçu aucune indemnité; elle a pourtant perdu son mari, vers 1618 : il a été " tué et mis en pièce en la montagne du Chasteau Lambert ", laissant trois petits enfants. Barbe se résigne donc dix-huit ans plus tard à solliciter " un droit de myne pour l'ayder a entretenir sur sa viellesse ». Pour obtenir une aide financière, la veuve doit toujours adresser une requête : "Remonstre humblement Elisabeht Lorentz [déjà rencontrée ci-dessus] qu'il y a environ huit ans [donc vers 1622] que son mary fut tué d'un coup de pétard dans la montagne et qu'il luy a laissez ung pauvre enfant lequel est inocent d'esprit. " Elle demande une aumône pour nourrir

15. PiPOnNiER, Françoise et Mane, Perrine, Se vêtir au Moyen Âge, Paris, Adam Biro, 1995, p. 59-69. Pour ce paragraphe, encore plus que pour le reste du texte, j'ai bénéficié des compétences de la médiéviste Laurence DELOBETTE : qu'elle en soit remerciée. 
cet enfant et elle-même, " n'ayant aulcung aultres moyens que ce qu'elle gaigne a bouquer [voir la remarque ci-dessus] quelque peu ce faisant elle priera Dieu pour la prosperitez de la montagne ", c'est-à-dire des mines. On finit par lui accorder, à elle et à sa sœur Anne, deux francs par mois, mais " sans espoir d'y plus retourner ${ }^{16}$ ".

Entre Villersexel et Héricourt, la saline de Saulnot approvisionne le comté de Montbéliard. Étant donné la raréfaction du bois, et son prix croissant, d'autres combustibles sont recherchés, notamment la tourbe et le charbon de terre. Vers 1590, on découvre de la houille « en la montagne de Haussemont, finaige de Corseille ", hameau voisin de Saulnot. Cette mine est aussitôt exploitée : elle emploie dix-neuf mineurs en 1594 et vingt-sept en 1595. Trois galeries y sont creusées, avant 1606. Puis l'extraction diminue et on ne compte plus que trois mineurs en 1614. Les beaux registres de comptes de la seigneurie de Granges mentionnent une distribution d'argent et de blé faite aux mères, aux femmes et aux sœurs d'ouvriers "suffoqués par le mauvais air " dans ces "houillères d'Assemont ". Probablement faut-il comprendre qu'une sorte de " coup de grisou " a causé des victimes parmi les mineurs. On déplore notamment la mort de Nicolas Boley, fils de Jeannette, veuve de Jean Boley, le 4 janvier $1616^{17}$. On est donc loin de la présentation idyllique des mines de la Renaissance exposée ci-dessus.

Quoi qu'il en soit, les femmes employées sur les sites miniers sont peu nombreuses. Pour saisir la vie des ouvrières, nous disposons d'une source beaucoup plus abondante, celle des salines de Salins, dans le massif du Jura.

\section{Les ouvrières des salines}

Salins est un des plus grands centres industriels d'Europe depuis des siècles ${ }^{18}$. Avant d'évoquer les femmes qui y travaillent, une présentation de ce centre industriel est indispensable.

\section{Le fonctionnement des salines et leur main-d'œuvre}

Plusieurs sources d'eau très fortement salée sont exploitées depuis la Préhistoire, très exactement à partir de l'année 3334 avant Jésus-Christ selon les derniers résultats des études archéologiques menées sur place.

16. Toutes ces requêtes sont tirées des Arch. dép. du Doubs : B 238; B 1370; B 1374; B 1468.

17. Arch. dép. de la Haute-Saône, E 159; cf. BouvarD, André, « Heinrich Schickhardt, technicien des salines. Les techniques de fabrication du sel vers 1600. Les salines de Salins et de Saulnot à la fin du Xvi siècle ", Mémoires de la Société d'Émulation de Montbéliard, 1983, n 106, p. 55-115.

18. DeLSALLE, Paul, « Paisaje industrial y region industrial en Europa en los siglos XVI, XVII y XVIII ", Revista de Historia Industrial, Barcelona, n ${ }^{\circ}$ 14, Año 1998, p. 173-187; La France industrielle aux XVe, XVIT, XVII ${ }^{e}$ siècles, Paris, Ophrys, 1993; «Images et réalités du paysage industriel aux XVI et ${ }^{\mathrm{e} V I I}{ }^{\mathrm{e}}$ siècles ", Les paysages à l'époque moderne, Paris, Presses de l'Université de Paris-Sorbonne, 2005, (à paraître). 
Au Moyen Âge, Salins est devenu « un complexe industriel parmi les plus imposants d'Europe ${ }^{19}$ ", produisant environ 7000 tonnes de sel par an (en 1467), soit 20 tonnes par jour ${ }^{20}$. Les chiffres du début du XVII ${ }^{\mathrm{e}}$ siècle sont plus modestes, témoignant d'un déclin progressif : 2047 tonnes en 1632, soit environ 5,6 tonnes par jour. La baisse s'expliquerait par la chute de la salinité $\left(20 \%\right.$ au Xve $^{\mathrm{e}}$ siècle contre $10 \%$ au XvIII $^{\mathrm{e}}$ siècle), ce qui nécessite aussi beaucoup plus de chauffage et donc de bois.

Trois entreprises se partagent l'exploitation. La première est la Grande Saunerie ou saunerie du Bourg-Dessus. Depuis le xiIle siècle, elle appartient à la famille de Chalon. Par héritage et confiscation, elle passe ensuite aux Habsbourg, devenus comtes de Bourgogne, jusqu'en 1678 au moment où Louis XIV s'en empare. Cette entreprise forme une source de profit considérable. La comptabilité du comté de Bourgogne, pour l'année 1590, inscrit le profit de 114000 livres pour les salines, ce qui représente $60 \%$ des recettes domaniales ${ }^{21}$. La deuxième entreprise, appelée la chauderette de Rosières, n'est qu'une annexe de la Grande Saunerie. Enfin, la troisième s'appelle la Saunerie du Bourg-Dessous ou puits à muire, et elle appartient collectivement à un groupe de rentiers (ecclésiastiques, nobles, bourgeois) mais techniquement elle est aussi une dépendance de la Grande Saunerie. Les archives de cette dernière saline nous fournissent beaucoup d'informations sur les ouvrières ${ }^{22}$.

Les sources d'eau salée sont captées par des puits, creusés au Moyen Âge, afin d'obtenir une plus forte salinité. Une immense galerie, véritable cathédrale souterraine, permet la communication entre toutes les installations ${ }^{23}$. Des machines permettent ensuite de remonter l'eau salée (la " muire ") en surface, à l'aide de chaînes sans fin de barils ou de tonnelets, appelées "noria ", " signole " ou encore " paternoster " puisque ce système rappelle celui d'un chapelet. Les roues sont actionnées par des manèges à chevaux, puis, vers 1750, par la force hydraulique, malheureusement moins efficace en période d'étiage car le torrent (la Furieuse) qui longe l'usine a un débit très irrégulier.

L'ensemble formé par les salines a donné naissance à une vraie ville, peuplée de 1235 feux $^{24}$ en 1614, soit 6000 à 7000 habitants, et très fortifiée. Les deux anciens bourgs médiévaux ont été réunis. On y compte quatre églises paroissiales, dont deux sont en même temps des collégiales, une

19. L'expression est de BERGIER, Jean-François, Une histoire du sel, Paris, PUF, 1982, p. 82.

20. Les salines de Salins au XIII siècle, cartulaire et livre des rentiers, édité par René LOCATELL, Denis Brun, Henri DuBors, Besançon, Annales littéraires de l'Université, 1991, p. 28-48.

21. GRISEL, Denis et GuYARD, Patricia, Trésor des chartes des comtes de Bourgogne et chambre des comptes de Dole; documents sur l'administration du domaine et la féodalité du comté de Bourgogne (XIII -XVIII siècle), Besançon, Archives départementales du Doubs, 2000, p. 20-30.

22. Archivo General de Simancas, Estado 528 (1), 1253 (18), 2167 (33); Secretarias provinciales 2558 (74-128), 2562 (13), 2567 (5); Arch. dép. du Jura, série A, fonds des salines de Salins.

23. Bibliothèque d'étude et de conservation, Besançon, collection Chifflet, Ms 44, $\mathrm{f}^{\circ} 71$; dessin d'Anatoile Chastel.

24. Arch. dép. du Doubs, 2 B270. 
autre église collégiale, plusieurs hôpitaux et de nombreux couvents. Sur les sommets voisins, des forteresses surveillent et protègent la saline : Châtelbelin, Châtelguyon, Bracon ${ }^{25}$. Du haut de ces forts, sans doute voiton en permanence la fumée et la vapeur d'eau qui s'élèvent des chaudières d'évaporation de la muire. L'usine est elle-même une petite cité dans la ville, avec ses propres murailles, sévèrement gardées. On y trouve toutes les installations, les ateliers ou ouvroirs appelés "bernes " avec les chaudières pour l'évaporation de la muire remontée à la surface, mais aussi des forges et de grands entrepôts pour le bois et le sel ${ }^{26}$.

Dans cet ensemble monumental, la main-d'œuvre est plus nombreuse qu'on ne l'a dit. Pour la période médiévale, le nombre total est inconnu; nous savons simplement que la grande saunerie emploierait 80 ouvriers en $1475^{27}$. Or, le $\mathrm{XV}^{\mathrm{e}}$ siècle correspond à l'apogée de la saline; ce nombre très faible ne traduit sans doute qu'une partie de l'effectif. D'ailleurs, un document du début du XVII ${ }^{\mathrm{e}}$ siècle parle d'environ 800 personnes ${ }^{28}$. Un autre relevé détaillé de la main-d'œuvre employée dans chaque atelier aboutit au nombre de 1040 personnes ${ }^{29}$. Encore ne s'agit-il là que du personnel interne, principalement ${ }^{30}$. En effet, la saline utilise encore les services d'une abondante main-d'œuvre à l'extérieur. Pour chauffer l'eau salée et la faire évaporer, une grande quantité de bois calibré est nécessaire : " tant de bois, que l'on ameigne tous les jours, que c'est chose merveilleuze " écrit Philippe de Vigneulles, bourgeois de Metz, pèlerin se rendant à Saint-Claude en $1512^{31}$. Toute la région est donc marquée par l'industrie de Salins, très xylophage ${ }^{32}$. Un contrat est signé pour la fourniture de 80 millions de fagots en $1624^{33}$. On estime la consommation

25. Cf. le plan de Salins publié par Delsalle, Paul, La Franche-Comté au temps de Charles Quint, documents choisis et présentés, Besançon, Presses universitaires franc-comtoises, 2000 , p. 26.

26. On retrouve un système comparable dans d'autres salines d'Europe; cf. HoCQUET, Jean-Claude "L'histoire de la production du sel dans les pays allemands au Moyen Âge ", Bulletin de la Mission historique française en Allemagne, 1999.

27. PRINET, Max, "Les ouvriers des anciennes salines franc-comtoises ", Les annales franc-comtoises, 1897, p. 271-276; cf. aussi DuBoIs, Henri, " À Salins au XV siècle : problèmes humains et problèmes industriels d'une entreprise ", Wirtschaftskräfte und Wirtschaftswege, (Festschrift für Hermann Kellebenz), Bamberg, 1978, p. 203-218.

28. Bibliothèque d'étude et de conservation, Besançon, collection Chifflet, $\mathrm{Ms} 44, \mathrm{f}^{\circ} 67$ et $\mathrm{f}^{\circ} 114$.

29. Idem, $\mathrm{f}^{\circ} 68$ et $\mathrm{f}^{\circ} 115$. Il faut tenir compte du fait que la saline travaille jour et nuit; les effectifs sont d'autant plus considérables. On ignore le nombre d'heures effectuées par chaque catégorie d'ouvriers et d'ouvrières.

30 . En règle générale, le traitement des affaires n'a pas lieu là où est produit le sel mais dans la ville la plus proche; Salins offre un cas différent, exceptionnel, probablement unique, selon BERGIER, Jean-François, Une histoire du sel..., op. cit., p. 164.

31. Chronique publiée par Jules GAUTHIER, Annuaire du Doubs, 1898, p. 40-44.

32. Cf. l'étude de GuYARD, Patricia, "Exploitation et réglementation des forêts autour de la grande saunerie de Salins. Contribution à l'histoire forestière comtoise (1470-1570) ", dans Delsalle, Paul et Delobette, Laurence (dir.), La Franche-Comté à la charnière du Moyen Âge et de la Renaissance, Actes du Colloque de Besançon, Presses universitaires de Franche-Comté, 2003, p. 283-312.

33. Arch. dép. du Doubs, B 2112. 
à 35000 stères au XVII $^{\mathrm{e}}$ siècle (cinq fois plus qu'une verrerie au XVIII ${ }^{\mathrm{e}}$ siècle), ce qui suppose en permanence l'exploitation forestière de 6000 hectares, dans un système à très courte révolution, donc en taillis ${ }^{34}$. Pour cela, tous les bois situés à trois lieues à la ronde sont réservés à Salins (il s'agit de la lieue comtoise, longue de $5,86 \mathrm{~km}$ ), soit un paysage transformé par les bûcherons à $18 \mathrm{~km}$ à la ronde. Des dizaines de communautés rurales sont mobilisées pour l'abattage et le transport du bois ${ }^{35}$. Plusieurs forêts sont entièrement gérées pour les salines. En réalité, les bois peuvent venir de quatre, cinq et même parfois de six lieues (soit $35 \mathrm{~km}$ ) ${ }^{36}$. Tout cela nécessite une main-d'œuvre considérable, en partie féminine.

Les femmes sont employées dans cette saline, non seulement à l'extérieur pour la collecte du bois mais aussi à l'intérieur pour la confection des pains de sel. Or, curieusement, les très nombreux travaux historiques sur les salines de Salins n'en parlent pas ou très peu; tout au plus mentionnentils la présence d'ouvrières mais sans s'y attarder davantage ${ }^{37}$. Dans l'iconographie salinoise, les femmes ne sont pas représentées. Les aquarelles de la fin du XVI ${ }^{\mathrm{e}}$ siècle et les gravures du début du XVII ${ }^{\mathrm{e}}$ siècle accordent une place aux hommes à plusieurs reprises mais jamais aux femmes ${ }^{38}$. Pourtant, les archives révèlent des effectifs féminins considérables et méritent qu'on s'y intéresse de plus près ${ }^{39}$.

34. Arch. dép. du Doubs, B 1812.

35. Archives générales du Royaume de Belgique, Bruxelles, Conseil des finances, ${ }^{\circ} 429$.

36 . Arch. dép. du Jura, A 18 : " pour le faict de contraindre les charretiers tant dudit Salins que a quatre lieues alentour dudit lieu de charroier bois en ladite saulnerie quatre jours la sepmaine si plus ne peuvent " (Salins, 1577); Arch. dép. du Doubs, B 239 à B 444; " quatre à cinq lieues à la ronde " (en 1601) d'après HAMMERER, André, Sur les chemins du sel. Activité commerciale des sauneries de Salins du XIV au XVIf siècle, Besançon, Cêtre, 1984, p. 190.

37. Par exemple : Brelot, Claude-Isabelle, Locateldi, René, DeBARD, Jean-Marc, GRESSET, Maurice, Augustin, Jean-Marie, Un millénaire d'exploitation du sel en Franche-Comté : contribution à l'archéologie industrielle des salines de Salins (Jura), Besançon, CRDP, 1981; Augustin, Jean-Marie, "Administration et justice dans les salines de Salins sous les Habsbourg ", Le sel et son histoire, Publications Université Nancy II, 1981, p. 289-318. BERGIER, Jean-François, Une histoire du sel..., op. cit., p. 194, a noté l'importance des femmes à Salins, en observant que le travail féminin caractérisait « les salines de Bourgogne, de Pologne et surtout d'Asie" ; cf. aussi Hocquet, Jean-Claude, Le sel et le pouvoir de l'an mil à la Révolution française, Paris, Albin Michel, 1985, p. 52-54 : l'auteur parle de " gynécée industriel ".

38. Hauptstaatsarchiv Stuttgart, N 220, T 59; le dessin est reproduit par BouvARD, André, "Un ingénieur à Montbéliard, Henrich Schickhardt, dessins et réalisations techniques (1593-1608) ", Société d'Émulation de Montbéliard, Bulletin et Mémoires, $\mathrm{n}^{\circ}$ 123, p. 20; Bibliothèque d'étude et de conservation, Besançon : collection Chifflet, ms $44, \mathrm{f}^{\circ} 71$; un des dessins est reproduit dans Simonin, Olivier, « Entre sel et terre : la Grande Saunerie de Salins-les-Bains, archéologie et histoire ", Éclats d'histoire, Besançon, Cêtre, 1995, p. 89; voir aussi Delsalle, Paul, La Franche-Comté au temps..., op. cit., p. 258; du même auteur : Les Franc-Comtoises à la Renaissance, Saint-Cyr, Sutton, 2005, p. 114-125.

39. WEBER, Renate, "Von " nassen und truckenen Dirnen ». Frauenarbeit in den bayerischen Salinen ", Salz macht Geschichte. Aufsätze..., op. cit., p. 142-147; il y a 33 femmes en 1581 à Reichenhall dans les chambres d'évaporation (3 à 5 femmes par chambre). 
Autour de la saline, il y aurait en effet « 3000 à 4000 ouvriers et ouvrières " selon un document daté de 1570 :

"Le nombre des coppans, chargeans et enlevans lesdictz bois [...] est si grant qu'il exede a plus de trois a quatre mil personnes ", tant hommes que femmes puisqu'il ajoute : " si fort addonnees ausdictz coppaiges et vendaiges de bois qu'ilz en delaissent toutes aultres entreprises si avant que l'on ne peult retreuver audict lieu de Salins ouvriers et ouvrieres a journees pour besongner es vignes ${ }^{40}$."

Parmi cette main-d'œuvre et ces travaux, les femmes ne se contentent pas de ramasser du bois; on les voit aussi conduire les véhicules. Ainsi, le 19 juillet 1607, un forestier rencontre la femme de Bon Guignard, demeurant à Dournon, " sortant du bois du Jurat, conduisant un charyot attelé de deux juments chargé d'ung pied de bois de sapin ${ }^{41}$ ». Les filles aussi sont à l'œuvre. En 1562, Loys Bassault, de Bartherans, bat et outrage " une fille conduisant bois pour ladite saulnerie, jusques à grande effusion de sang ". Les femmes sont également présentes à l'intérieur de la saline, ce qui n'est pas le cas dans d'autres usines, par exemple dans les salines de l'Arbonne, dans les Alpes, près de Bourg-Saint-Maurice ${ }^{42}$. A Salins, en 1466, il y a " des officiers, ouvriers et ouvrieres ${ }^{43}$ ".

Pour clarifier les choses et tenter de comprendre concrètement ce que font ces femmes, nous devons rassembler les différentes informations très dispersées, parfois contradictoires, toujours incomplètes ${ }^{44}$. La grande saline compte huit " bernes " ou ateliers; à côté, se trouvent les cinq ateliers du puits à muire, appelé aussi " Bourg Dessous ". Chacune de ces bernes (ou ateliers) de la grande et de la petite saline de Salins emploie plusieurs dizaines d'ouvrières. Parmi ces dernières, celles qui exercent les fonctions les plus importantes sont désignées par des noms curieux, plus ou moins issus du latin ${ }^{45}$.

Les "mettari " (métarry, mettarix, metatrix) ramassent le sel après l'évaporation de l'eau et remplissent les moules, les écuelles. D'où vient ce terme? On songe à " metator, oris " : qui mesure, qui jalonne. On peut penser aussi à l'italien " mestare ", remuer. Loys Gollut nous dit : " quatre femmes besongnent deux à deux à retour; l'une appellée Métarry (comme

40. Arch. dép. du Doubs, 2 B $2247, f^{\circ} 27$; «ilz se y retreuvent et assemblent par quatre vingt cent et quelques fois plus".

41. Arch. dép. du Jura, A 166, $\mathrm{f}^{\circ} 10 \mathrm{v}^{\circ}$.

42. HuDRY, Marius, "Les salines de l'Arbonne ", Métiers et industrie en Savoie, Annecy, Académie salésienne, 1974, p. 129-138.

43. Arch. dép. du Doubs, B 187.

44. DelSALle, Paul, " Les ouvrières des salines de Salins (Jura), XVe-xvII" ${ }^{\mathrm{e}}$ siècles ", Histoire, économie et société, 2006, $\mathrm{n}^{\circ}$ 1, p. 15-31.

45. On retrouve des dénominations comparables dans l'Antiquité tardive, parmi les esclaves qui travaillent dans le secteur de l'habillement, par exemple le textor, la textrix (tisserand, tisserande), le sarcinator, la sarcinatrix (couturier, couturière); cf. MORABITO, Marcel, Les réalités de l'esclavage d'après le Digeste, Annales littéraires de l'Université de Besançon, 1981, p. 87. Ce Digeste est une compilation juridique de l'époque de Justinien (534). 
metatrix), qui est celle qui prend le sel dedans sa mesure, et forme le salé ou salignon (= le pain de sel), le faisant moindre d'un quart à cinquième que celui du puits à muire ${ }^{46}$, et plus petit d'un tiers que celuy de gros sel chauderette". Il ajoute :

«Et convient notter, ce que nous avons leü entre les philosophes, que le sel ne se façoneroit si Métarry ne l'humectoit d'eau salée, qui provient de la distillation cheante de la sille [plan incliné pour égoutter], qui est propre (pour estre desjà cuitte) à ce que le sel se puisse serrer, ce qu'une autre eau ne pourroit faire. Métarry haïant formé le salignon, et tiré de l'escuelle, le pose sur le brasier estendu par la sille ${ }^{47}$."

Le 23 juin 1523, une "mettary " qui a été expulsée pendant la peste et qui se retrouve désormais sans ouvrage reçoit une aide ${ }^{48}$. Philiberte Fatton, Perreinin Bernard, Margueritte Vaulcher ou Vaulchet, Parise Bonnet ou encore Françoise Boisset sont des mettaris en $1620^{49}$.

Les « fassari " (fassary, fassarix, factrix) façonnent les pains de sel, appelés aussi " salignons " : "L'autre femme est appelée Fassary (comme factrix) est celle qui façone et done le tour au salignon ${ }^{50}$. " Faut-il voir ici un dérivé de " factor, oris " signifiant fabricant? Max Prinet a décrit ce travail : " La fassari pétrissait le sel à l'aide de la muire de l'auge, sur un bloc de bois ou massou, le régularisait avec la main, et renversait l'écuelle dans une autre, plus grande, garnie d'une couche de sel en grains pour empêcher l'adhérence. Le pain ou salignon ainsi formé était replacé sur la sille", sorte de plan incliné pour égoutter ${ }^{51}$. Antoinette Malcourant obtient une charge de " fassarix en la berne de Chatelain, 18 juillet $1530^{52}$ ». Jeanne Jannain, Barbe Bault, Philiberte Borde, Nicole Clerc comme Guyette Peleteret appartiennent au groupe des fassaris en 1620.

Les "sechari " (séchary, secharry, seicharix) font sécher les pains de $\operatorname{sel}^{53}$ : " la femme nommée Sécharry prend [le salignon] après 24 heures, combien que le sel qui est au bas de la sille est plus difficile à cuire. Et de

46. Ce qui signifie que la dimension des pains de sel n'est pas uniforme, ceux de la grande saunerie étant plus petits que ceux du puits à muire et de la Chauderette.

47. Gollut, Loys, Les mémoires historiques..., op. cit., col. 136 à 182.

48. Arch. dép. du Doubs, B 189.

49. Bibliothèque d'étude et de conservation, Besançon, collection Chifflet, ms 44, $\mathrm{f}^{\circ} 147$.

50. Gollut, Loys, Les mémoires historiques..., op. cit., col. 136 à 182.

51. PRINET, Max "L'industrie du sel en Franche-Comté avant la conquête française ", Mémoires de la Société d'Émulation du Doubs, 1897, p. 61-216. Il se fonde notamment, aux Arch. dép. du Doubs, sur le dossier coté B207, expérience du 26 mars 1537 (n. st. 1538); du même auteur : "Les ouvriers des anciennes salines franc-comtoises ", Les Annales franc-comtoises, 1897, p. 271-276.

52. Arch. dép. du Doubs, B 191. Document incommunicable; l'information est donc tirée du répertoire. Le dépouillement systématique des registres de délibérations aurait sans doute apporté d'autres détails sur ces ouvrières.

53. Ce travail est attesté aussi dès 1351 à la saline de Grozon, près de Poligny; les femmes sont payées 4 deniers estevenants, c'est-à-dire qu'elles reçoivent le même paiement que les "berniers qui governent une berne "; Arch. dép. du Doubs, B 309. 
ce, elle est appelée Sécharry, pour ce qu'elle manie le saligon seiché ${ }^{54}$ ». Il s'agit d'un travail très délicat car il faut éviter de brûler le sel. Guillemette Gorin, Catherine Jannain, Catherine Fyot, Loyse Boisset, parmi d'autres, assurent cette fonction en 1620 .

Les " portarri " (portarry, portarix, portatrix) portent, probablement. Le terme viendrait-il de "portator, oris " à traduire par porteur? Cette fonction se rencontre au "Puits à muyre " ou "Bourg Dessoubz " au milieu du $\mathrm{XVI}^{\mathrm{e}}$ siècle. Les portarix apparaissent peu et nous ne savons pas exactement quelle est leur tâche.

Les " estagnarri " (estagnary, esteignarix, estaignarrix, esteignary, extinctrix) doivent peut- être réguler le feu, à partir des braises : "Une autre femme, dite Eteignari était chargée de jeter de l'eau sur cette braise pour l'éteindre et ménager ainsi du charbon pour le séchage du sel ${ }^{55}$. " En 1620, Marthe Michiel, Jehannette de la Tour, Alix Marguet ${ }^{56}$, Jeanne Fertey, Jeannette Richard, Jeanne Menestrier, Jehanne Aviet et Phelippa Boisset assurent ce travail.

Enfin, les «tirarii " (tiraris, tirarry) retirent les braises, à l'aide de râbles de fer : " La braise qui tombait au travers de la grille, était extraite du fourneau par une femme nommée la Tirari de feu, qui la prenait sur une longue pelle appelée épi ${ }^{57}$. "Les braises récupérées servent à sécher les pains de sel ${ }^{58}$. Catherine Thomet, Blanchon More, Thoinette Boussu ou Bonssu, Jeanne Barbet, Thienette Billard, Claude Brung, ou encore Clauda Thomet, exercent cette fonction en $1620^{59}$.

Quoi qu'il en soit exactement de ces travaux mal connus, il s'agit toujours de tâches très spécialisées confiées à des femmes, ce qui n'est pas si fréquent à cette époque. Il n'est sûrement pas inutile d'insister au passage sur la division du travail constatée ici. La forme de production est très éloignée des archaïsmes constatés dans les salines de Bavière, dans lesquelles des femmes sont exploitées en tant que force motrice. Ainsi, à Hallein, la noria est actionnée par une roue ou " cage d'écureuil ", elle-même mue par la force des femmes qui marchent à l'intérieur à longueur de journée, et non par des chevaux ou autres animaux ${ }^{60}$.

54. Gollut, Loys, Les mémoires historiques..., op. cit., col. 136 à 182.

55. Prinet, Max, "L'industrie du sel en Franche-Comté... ", article cité, p. 193.

56. Archives générales du royaume de Belgique, Conseil des finances, 69; voir sa requête en fin d'article.

57. Prinet, Max, "L'industrie du sel en Franche-Comté... ", article cité, p. 201.

58. Belhoste, Jean-François, La métallurgie comtoise, XVe-XIXe siècles. Étude du val de Saône, Besançon, Inventaire général, 1994, p. 41.

59. Bibliothèque d'étude et de conservation, Besançon, collection Chifflet, ms 44, $\mathrm{f}^{\circ}$ 144-149.

60. WeBER, Renate, "Von "nassen und truckenen Dirnen ". Frauenarbeit in den bayerischen Salinen ", Salz macht Geschichte. Aufsätze, Herausgegeben von Manfred Treml, Wolfgang Jahn und Evamaria Brockhoff, Augsburg, Haus der Bayerischen Geschichte, p. 142-147. 
Ces tâches sont désignées dans les textes comme des "charges ", des " états " ou même des " offices", viagers ${ }^{61}$, et transmissibles à titre héréditaire de mère en fille, parfois de tante en nièce ${ }^{62}$. De nombreuses traces attestent de cette situation, totalement insoupçonnée jusqu'à maintenant. Le système est assez simple : une femme en fonction propose une autre femme pour lui succéder. Elle doit être digne de confiance, bien sûr. Lorsque celle-là est acceptée par le conseil de la saline, elle est instituée officiellement et prête le serment sur la Bible de travailler dans le respect des engagements, des pratiques, des habitudes ${ }^{63}$. La succession ne prend effet qu'à la mort ou à la démission de la titulaire de la fonction. Voici, par exemple, Jehanne Bondier, épouse de Pierre Carerroz, qui est fassary en la berne de Balerne; en 1548, elle se désiste en faveur de sa fille Regnaulde Carerroz $^{64}$. Une autre femme, Perrenette Rousselet, "vesve de feu Loys Naudin ", est " estaignarrix en la berne de Bernette ». En 1550, elle annonce que " de long temps elle a desservi ledit estat bien et dehuement sans y avoir fait faulte et le grand eaige ou elle est constituée requerant dois [=dès] maintenant pour l'advenir pourveoir et instituer audit estat d'estaignarix Françoise Nauldin sa fille a condition qu'elle ladite Perrenette jouyira des gaiges et prouffitz dudit estat sa vie naturelle durant ${ }^{65}$ ". Elle s'assure donc une future pension.

En 1570, Girarde Odet se sent aussi trop vieille pour exercer sa charge de " portarrix de muyre en la berne de Balerne ", ce qu'elle fait depuis plus de cinquante ans! Elle est déjà veuve de " feu Estienne de Ban ». Maintenant, comme elle " se sentoit vielle et caducque ${ }^{66}$ ", selon les propos de son procureur (Étienne Girardot), elle remet sa charge en demandant de l'offrir à la demoiselle Claude Alix. Or, celle-ci n'est autre que la fille de Maître Claude

61. Il s'agit de " véritables charges viagères " écrit Max PRINET, dans " Les ouvriers des anciennes salines ... ", article cité, p. 272; l'office est défini comme "nomination à vie ", selon Doyle, William, La vénalité, Paris, PUF, 2000, p. 24.

62. Pour les employés masculins aussi, il existe toute une série d'offices, dès le xIV siècle : maîtres moutiers, gardiens de source, portiers, clercs, payeurs, vendeurs, taxeurs de bois, forestiers, graites, bénatiers, fèvres, etc.; $c f$. les nombreuses ordonnances sur ce point, Arch. dép. du Doubs, B 290 à B302. L'office de fèvre est transmissible, en 1453; Arch. dép. du Doubs, B 290. Des offices sont vendus : vente d'un quart d'office de bénatier (à la berne de Glapin) cédé par Jean Prévostet à Estevenin Certain, pour 22 florins en 1492; Arch. dép. du Doubs, B 290. Ici, l'office est confirmé par un acte appelé "commission". Philippe II accorde l'hérédité des offices de fèvres employés à l'entretien des chaudières, de mâle en mâle, attendu l'importance d'ouvriers expérimentés et leur rareté à la suite des pestes : en 1587, il ne reste plus à la saunerie " que deux entenduz en tel art et mestier, l'ung d'eux eagé d'environ quattre vingts ans "; Arch. dép. du Doubs, B 294.

63. La prestation de serment est renouvelée chaque année, selon le témoignage d'Anthoine (sic) Carrietty, épouse de Jean Boissia, en 1630; Arch. dép. du Doubs, 2 B 3341.

64. Arch. dép. du Jura, A 17, pièce n ${ }^{\circ}$ 15. Document transcrit par Anne Bouchy dans le cadre de mon cours de paléographie.

65. Arch. dép. du Jura, A 17 , pièce $n^{\circ} 3$.

66. La vieillesse et la caducité des ouvrières sont souvent mentionnées. C'est par exemple le cas de Ysabel Vernerey, en 1577; Arch. dép. du Jura, A 17, pièce ${ }^{\circ} 3$ : " elle se santoit fort caducque". 
Alix, bourgeois de Salins et conseiller du puits, un des responsables de la saline ${ }^{67}$, c'est dire, une fois de plus, que les ouvrières ou manœuvres, n'appartiennent pas nécessairement à la couche la plus pauvre de la société salinoise ${ }^{68}$.

Jehanne Vuillemol succède, avant 1577, à Catherine Duffour, sa mère, laquelle a exercé très longtemps, peut-être pendant cinquante ans (elle aussi!) la charge de "sécharry " à Poupet. Jehanne demande ensuite que sa propre fille, Catherine Millet, lui succède. Nous avons donc ici trois générations attestées pour l'exercice de la même fonction ${ }^{69}$. Dernier exemple : Estienette Coquelin avait été " pourvue en l'état d'estegnarrix " pour remplacer plus tard sa mère Anthonia Picouteal, en 1577. Celle-là " estoit allee de vie a trepas ", Estienette Coquelin devient aussitôt " estagnery ", en 1586.

Serait-il aussi possible de suivre la carrière professionnelle de certaines ouvrières, si tant est qu'il y en ait une? Anthoina Bersot, ou Bersault, épouse de Nicolas Bonnot, succède à Anatholia Galiod en tant que tirary de sel en la berne de Balerne, en 1586. Treize ans plus tard, nous retrouvons une Bersot, mais Anthonia cette fois-ci, tirary de sel à Balerne, qui ne peut plus exercer cette charge personnellement et présente à sa place Henriette (Henrietta?) Bornet ou Boquet, en $1599^{70}$. Les homonymies étant fréquentes, de même que les variantes orthographiques, il convient d'être prudent. Nous retrouvons, par exemple, une autre Anthonia Bersot, en 1586; mais elle est séchary en la berne de Bernette et son mari s'appelle Nicolas Bornet; en outre elle remplace Jehanne Cardon. Anthoina Bersot et Anthonia Bersot ne sont donc pas une seule et même personne ${ }^{71}$. Une véritable prosopographie est envisageable, qui utiliserait toutes les informations possibles sur chaque ouvrière, notamment celles issues des registres paroissiaux et des dossiers de la juridiction de la grande saunerie de Salins ${ }^{72}$. En attendant, il semblerait que les charges soient détenues à l'intérieur des mêmes familles.

Pour bénéficier d'une entrée en fonction dans l'une de ces charges à la saline, certaines ouvrières obtiennent le soutien de personnalités de premier plan ${ }^{73}$. Le 14 décembre 1524, Jean Carondelet, qui n'est autre que l'archevê-

67. Arch. dép. du Jura, A 17, pièce $n^{\circ} 7$.

68. Arch. dép. du Doubs, B 2073; en 1560 et 1572.

69. Arch. dép. du Jura, A 17 , pièce ${ }^{\circ} 5$.

70. Arch. dép. du Jura, A 17; acte du 29 décembre 1586; l'éventuel lien de parenté entre les deux femmes n'est pas mentionné; Arch. dép. du Jura, A 17, pièce $n^{\circ} 1$ : acte du 4 janvier 1599; le document précise que la personne pourvue de la charge doit exercer en personne (" en chef "), sinon présenter une femme idoine pour être pourvue à son tour.

71. Arch. dép. du Jura, A 17; acte du 30 décembre 1586.

72. Arch. dép. du Jura, A 163; minutes des jugements, de 1474 à 1698; journées de la justice de la grande saunerie, de 1601 à 1684 .

73. DelSAlle, Paul et Delobette, Laurence, "Les charges et les offices des ouvrières spé cialisées de Salins (XVe-XVIII siècles) ", Mémoires de la Société pour l'histoire du droit et des institutions des anciens pays bourguignons, comtois et romands (à paraître). 
que de Palerme, fidèle serviteur de Charles Quint, le prélat le plus puissant des Pays-Bas, président du Grand conseil de Malines et président du Conseil privé $^{74}$, adresse, depuis Bruxelles, une lettre de recommandation aux directeurs de la saline, en faveur de Jehanne Druhet qui est, dit-il, « une povre cousine ". Il espère qu'elle sera choisie pour succéder à sa défunte mère qui exerçait auparavant la même fonction ${ }^{75}$. Or, la veille, c'est la gouvernante ellemême, Marguerite d'Autriche, tante de l'Empereur, souveraine des Pays-Bas et de Franche-Comté, qui avait adressé aussi une lettre de recommandation aux administrateurs de la saline en faveur de cette ouvrière, sœur de son " bien aimé huissier ". Elle espère qu'ils reconnaîtront en Jehanne Druhet une personne "ydoine " pour exercer cette charge ${ }^{76}$. Il est donc évident que ces charges sont très recherchées et ne sont accordées qu'à des femmes de confiance choisies parmi les membres parfois " pauvres ${ }^{77}$ " de familles proches des maîtres de la saline. On peut supposer aussi que Jehanne Druhet a écrit (ou fait écrire) directement aux membres de sa famille présents à Malines et à Bruxelles pour obtenir l'appui décisif des autorités susceptibles d'influencer la décision finale des directeurs de la saline.

Toutes les indications concordent, montrant que ces offices sont transmis de façon héréditaire ${ }^{78}$. Toutefois, un témoignage indique que la charge n'est pas forcément accordée gratuitement. En 1630, Bonne Delezet, épouse de Huguenin Girard, est fassary depuis 35 ans; elle a " environ 50 ans". Elle exerce sa charge en l'ouvroir de Balerne et précise : " qu'il y a envyron quatorze ans [soit vers 1615-1616] qu'elle y achepta un office de la fille de Simard Galand mais il y a envyron trente ans qu'elle est fassary tant audit ouvroir qu'en d'aultre ${ }^{79}$ ". Autrement dit, une ouvrière peut acheter un office devenu vacant, transmis par une autre ouvrière mais nous ne savons pas si la somme est remise à la saline ou à l'ouvrière qui transmet sa charge. L'étude de la comptabilité ne nous permet pas, dans l'état actuel des recherches, de répondre à cette question. Toutefois, pourquoi acheter un office quand on occupe déjà la fonction? Probablement pour pouvoir le transmettre à son tour.

Ces fonctions, presque héréditaires, se rencontrent aussi chez les " gardes ", parmi lesquels, autre sujet d'étonnement, se trouvent aussi des femmes, comme l'écrit Loys Gollut à la fin du Xvi siècle :

«Mais par dessus les six, est une femme, appelée la garde, qui est tenuë de rendre compte et de faire former tout le sel envoḯ en l'ouvroir. Puis,

74. Voir la notice qui lui est consacrée dans Carolus, Charles Quint, 1500-1558, Gand, Snoeck-Ducaju \& Zoon, 2000, p. 198-199.

75. Arch. dép. du Jura, A 17; document transcrit par Caroline Godard, dans le cadre de mon cours de paléographie.

76. Arch. dép. du Jura, A 17.

77. Reste à connaître la réalité qui se cache derrière ce mot.

78. Sur l'hérédité de certaines charges, voir Maurice GRESSET « La situation avant la conquête française ", dans L'introduction à la vénalité des offices en Franche-Comté, 16921704, Annales littéraires de l'Université de Besançon, 1989, p. 20-23.

79. Arch. dép. du Doubs, 2 B 3341. 
quand le sel est formé, elle mande les maistres moustiers et clercs du sel pour en prendre le nombre et en faire la description, à fin qu'ilz les r'apportent un chasqu'un lundy au conseil, devant messieurs les conseilliers officiers et vendeurs ${ }^{80}$."

Le fait que ces femmes exercent cette fonction de garde n'est pas négligeable. On écrit trop souvent, sur la foi de sources littéraires ou juridiques, qu'elles ne recueillent alors aucune confiance. L'exemple de Salins, aussi exceptionnel soit-il, tend à démontrer le contraire. Une charge de garde à la saline de Salins, sans aucun doute le lieu le plus précieux, le mieux protégé et le mieux surveillé de toute la Franche-Comté, n'est concevable que pour une personne de très grande confiance.

Indéniablement, ces fonctions de garde, qui s'apparentent un peu à celles de contremaître, sont importantes. En 1630, Margueritte Boudot, âgée d'environ 52 ans, est garde au puits à muire du Bourg Dessous, en l'ouvroir de Balerne. Elle exerce ses fonctions depuis trente-deux ans. Quand on lui demande en quoi consiste sa charge, elle répond que " son devoir est d'empescher que les ouvriers [ sic; elle contrôle donc des hommes] travaillant audit puitz a muyre ne commectent abuz en leurs charges ». Doit-elle contrôler les fassarris? Elle " dict qu'ouy ». Dans l'exercice de sa charge, a-t-elle remarqué que les fassaris diminuaient la longueur des pains de sel? Elle rétorque: "Non, et quant lesdites fassaris font les salees moindre, ladite respondante est coustumée de les rompre. " Elle explique aussi que si les fassaris commettent des fautes, elle est chargée de les expulser, mais qu'elle-même et les autres employées sont sujettes à la même peine ${ }^{81}$. La mission de la garde correspond donc bien au contrôle du travail à l'intérieur d'un atelier.

Dans chaque ouvroir, les gardes bénéficient d'aides, qui sont souvent leurs filles : "Creulx : Damoiselle Claudine Gaigneffin, garde, et ses deux filles ses aides; Poupet : Damoiselle Estiennette Culier, garde, Margueritte sa fille son ayde; Anne Chisseret, garde, sa fille Anne son ayde. " Le cas de Margueritte Donner de Clairmont est plus surprenant : elle a pour aide " son mary ". Le titre de demoiselle accordé à ces femmes, et à certaines ouvrières dont nous avons parlé, n'est pas anodin. Il est habituellement réservé aux " femmes ou vesves de gentils-hommes ou nobles ou d'officiers principaux du souverain, ou de docteurs ès lois ou ès médecine ", selon l'édit du parlement de Dole publié le 20 juillet 1626.

La fonction de garde est, elle aussi, héréditaire. La "damoyselle" Marguerite Maginet, épouse de "l'honorable homme " Jehan Berchey, est "l'une de nos gardes ${ }^{82}$ " à la saline du puits à muire en 1548; elle a été admise dans cette fonction " puis environ dix-huit à vingt ans ", soit vers 1528-1530. Elle travaille en compagnie de sa fille, Claude Berchey, âgée de 14

80. Gollut, Loys, Les mémoires historiques..., op. cit., Dole, 1592, chapitres XXIII à XXXII, col. 136 à 182 .

81. Arch. dép. du Doubs, 2 B 3341

82. Ce sont les rentiers du puits à muire du Bourg-Dessous qui parlent; Arch. dép. du Jura, A 17, pièce 13 . 
ou 15 ans, " tant de jour que de nuit ${ }^{83}$ ". Marguerite exprime le souhait que, après sa mort, sa fille Claude lui succède. Cette dernière est acceptée par le conseil de la saline et prête serment sur la Bible. Vingt-quatre ans plus tard, Claude ou Clauda, qui a donc 38 ou 39 ans, est " garde en la berne de Bernette "; en 1572, elle est pourvue de cet " estat " du vivant de sa mère exerçant la même charge ("feu damoiselle Marguerite Maginet "). Aussitôt le trépas de sa mère, Clauda devrait prendre sa suite. Or, " elle ne pourroit exercer personnellement pour ce qu'elle est mariée au lieu de Poligny ", ville éloignée de Salins de plus de $20 \mathrm{~km}$. De ce fait, Clauda remet sa charge à la disposition du conseil de la saline, tout en proposant, comme elle en a le droit, une autre femme pour exercer cette fonction à sa place. Elle avance le nom de Jehanne Rutyet, épouse de Claude Brenet ${ }^{84}$.

Claudine Gorin est aussi " une de nos gardes en la berne dicte Balerne ", en 1568. Depuis plus de soixante ans, soit vers 1508, sa tante Jehanne Buchin exerce la même fonction, à laquelle elle a formé sa nièce, " avec toute loyaulté et sincérité sans avoir commis faulte digne de reprehention ». Claudine Gorin, qui a donc peut-être soixante-quinze ou quatre-vingts ans, propose d'instituer, après son propre décès, sa fille Maguerite Baudet (ou Boudet) ${ }^{85}$.

Faute de sources (registres paroissiaux, recensements, etc.) mais aussi de recherche prosopographique, nous ne savons pas encore qui sont ces femmes. Toutefois, lorsque certaines d'entre elles sont présentées, il est facile de s'apercevoir qu'elles appartiennent à l'élite locale. C'est ainsi qu'une garde nommée Margueritte Boudot n'est autre que la femme de Jacques Jannet, procureur postulant au bailliage de Salins. Thiennette Lardon est l'épouse de Jacques Perret, un orfèvre ${ }^{86}$.

\section{Les conditions de vie et de travail}

Les conditions de vie à l'intérieur de la saline s'apparentent aux « rigueurs du servage ", selon Max Prinet : "Les ouvriers y étaient astreints à garder toute leur vie l'office qu'ils avaient reçu. Il leur était interdit, sous peine d'emprisonnement, de changer de métier. Jour et nuit, ils devaient être présents à la saunerie, et cela même les dimanches et jours de fêtes. Ils étaient obligés de coucher dans l'usine, n'ayant pour reposer "qu'un peu d'estrain ou fueillie de bois sur la terre". Certains ouvriers se plaignent d'être "détenus comme prisonniers" En compensation, ils reçoivent chaque dimanche un gros pain de sel, et périodiquement du drap ${ }^{87}$."

83. Le travail de nuit est pratiqué aussi à Reichenhall; cf. "Von "nassen und truckenen Dirnen". Frauenarbeit in den bayerischen Salinen ", Salz macht Geschichte. Aufsätze... op. cit., p. 142-147.

84. Arch. dép. du Jura, A 17.

85. Arch. dép. du Jura, A 17.

86. Arch. dép. du Doubs, 2 B 3341; en 1630.

87. PRINET, Max, "Les ouvriers des anciennes salines ", article cité, p. 272; l'auteur ne précise pas ses sources. 
En réalité, cela ne concerne qu'un tout petit nombre d'ouvriers et peutêtre aucune ouvrière. D'ailleurs, on imagine mal le logement de 800 personnes dans l'usine! Il est toutefois certain qu'une partie de la main-d'œuvre loge dans l'enclos de la saline. Le vendredi 16 octobre 1562, Pierre Guyon injurie Clauda Galet, la veuve de Nicolas Viron, puis il lui jette des pierres au point de la blesser; le rapport précise bien que cette violence a été commise sur la personne de Clauda Galet " estant en ladite saulenie devant la maison ou elle faict sa résidence ${ }^{88}$ ". Certains responsables des salines habitent sur place avec leur famille. En 1624, Catherine Colin fait son testament " en la grande saulnerye d'illec ou ladite Damoiselle Colin fesoit sa demeurance $^{89}$ ". Une visite des édifices menée en 1651 mentionne aussi des "logements d'ouvriers ${ }^{90}$ ".

Les ouvrières apparaissent parfois collectivement. À l'époque de Charles Quint, en 1537, il est question d'une sorte de requête ou une pétition présentée par " des povres menuz ovriers et menues ovrières de la saunerie $^{91}$ ". Dans l'enceinte de l'usine, cette main-d'œuvre est soigneusement encadrée et contrôlée. Le 27 septembre 1537, on défend aux officiers, ouvrières et ouvrières " de parler du diable " sous peine d'une sévère prison ${ }^{92}$. Une " taverne à pain et à vin " est créée dans l'enceinte de l'usine, en 1534 , pour les ouvriers et ouvrières afin « qu'ils n'ayent occasion de traiger [= circuler] par la ville", principalement en temps de peste ${ }^{93}$. Des espaces de loisir ou de détente sont même installés à l'intérieur de la saline puisqu'on y trouve un jeu de paume, en $1623^{94}$. La saline dispose de sa propre juridiction, y compris la haute justice; des "journées " sont tenues régulièrement pour juger les voleurs et les querelleurs. Cette justice a surtout compétence pour le vaste ressort des bois qui alimentent les salines. Il y a même un pilori, bien attesté en 1531. Les femmes apparaissent peu souvent, sinon comme victimes, rarement responsables d'un méfait ${ }^{95}$.

Nous faisons plus ample connaissance avec certaines de ces ouvrières grâce à une enquête menée en 1629 au sujet de pains de sel qui sont confectionnés à l'aide d'écuelles non conformes. La suspicion règne dans

88. Arch. dép. du Jura, A 163. Cette question du logement des ouvrières n'a pas été étudiée; selon BERGIER, Jean-François, Une histoire du sel, op. cit., p. 196, "même à Salins ou à Hallein, la main-d'œuvre habite les environs plutôt que la cité elle-même. " A Salins, il me semble, dans l'attente d'une investigation sur ce point, qu'une grande partie de la main-d'œuvre vit dans la ville et le reste dans la saline.

89. Arch. dép. du Doubs, 2 B 638. Elle est l'épouse « du sieur Claude Perriet, co-admodiateur des saulneries".

90. Arch. dép. du Doubs, B 2083.

91. Arch. dép. du Doubs, B 209.

92. Arch. dép. du Doubs, B 189; B 192; B 209. Cette série de documents n'est plus consultable.

93. Arch. dép. du Doubs, B 191.

94. Arch. dép. du Doubs, B 2082.

95. Arch. dép. du Jura, A 163; en juin 1540, la servante d'un employé de la saunerie est emprisonnée pour avoir blessé une autre femme dans l'usine; la justice se tient en la tour des Rôles (en 1623) : Arch. dép. du Doubs, B 2082. 
l'usine et l'enquête vise à découvrir qui a fourni aux ouvrières les récipients incriminés. Au cours de son interrogatoire, Henriette Godard, âgée d'environ quarante ans, veuve de Jean Bricon dit Jancour, expose qu'elle " exerce l'office de seichary sont passéz sept à huit ans ". Elle affirme que c'est un certain Menetard qui a forcé des ouvrières à utiliser une écuelle : " et alors ledit Menetard sortit dudit ouvroir et en alla quere une qu'il donna à ladite Françoise Baisset, fassary, disant : "Tien, depesche toy de travailler avec ceste escuelle!" Surquoy elle repliqua que ladite escuelle n'estoit pas bonne et qu'elle n'en vouloit former du sel. " Cette enquête met en évidence la diversité et l'hétérogénéité des âges d'une grande partie de la maind'œuvre. En effet, d'autres ouvrières apparaissent, qui sont plutôt jeunes : Clauda Marmet, femme de François Marmier, a 26 ans. Catherine Grillet, épouse de Claude Basset, "fassary en l'ouvroir de Chastelain ", a 26 ans aussi. Colombe Servant, 23 ans, exerce "l'office de fassary pour Catherine Broye ", elle est donc remplaçante, comme Danielle Rabyet, "mettary au lieu de Chrestienne Passoy en la berne de Beauregard " qui est encore plus jeune : 20 ans, le même âge que "L'Estiennette Carerroz ", " secharry " également. Certaines ouvrières sont plus âgées. Guyette Peletteret, " fassary en la berne de Chastelain ", a 35 ans. Françoise Basset, 41 ans, est déjà veuve de François Pelot. Françoise Godard, femme de Claude Grandjean, « depose que dois [= depuis] sa jeunesse elle a travaillé sans discontinuation ausdites saulneries". Or, comme elle est âgée de 60 ans, on peut en déduire qu'elle travaille dans cette entreprise depuis 45 ans, pour le moins ${ }^{96}$.

En 1630, plusieurs ouvrières sont accusées de malversation car les mâ̂tres de la saline ont constaté qu'il manquait " deux doigts " sur la longueur des " bénates ", les pains de sel. Les interrogatoires mettent en évidence la vie quotidienne de ces femmes et leur labeur, encore plus pénible lorsqu'elles travaillent de nuit ${ }^{97}$. Bonne Delezet, qui est fassary à Balerne, raconte que la confection des pains de sel aboutit à des résultats qui peuvent varier qualitativement; par exemple « le seel de remandure est ordinairement plus moitte ". Elle explique qu'en séchant le sel peut se réduire plus ou moins. Quand on lui demande si le même phénomène se produit dans tous les ateliers, elle réplique « qu'elle n'y vad rien en qu'elle a assez affaire de se mesler du sien »! Elle précise aussitôt " qu'elle fait son devoir et qu'elle ne gagne

96. Arch. dép. du Doubs, 2 B 624.

97. La saline travaille durant la nuit : "L'on ne se doit treuver de nuict à l'entour des bernes dudit puyts [...] pendant que l'on y fait les cuittes et que l'on y forme lesdits sels " (ordonnance du parlement, 17 août 1550). "Pourront neantmoins les habitans dudit Salins aller, venir, passer, et repasser de nuict par les rues estans à l'entour des bernes et ouvreurs du puys à muyre, pourveu qu'ils portent lumiere et qu'ils n'entrent dedans lesdites bernes et ouvreurs, saufs et reservé ceux qui ont charge et office " (idem). " Est interdit, et defendu à tous, tant en general qu'en particulier, de prendre, amener, ny apporter vendre esdites saulneries en la ville, ou fauxbourgs dudit Salins sur le col, teste, ou bras, aucuns bois de fassures par fardeaux, ou autrement, et à tous de les acheter, et aux charetiers, et autres amenans bois esdites saulneries de les prendre et amener " (1573 ou 1592); cf. Petremand, Jean, Recueil des ordonnances et edictz de la Franche-Comté de Bourgongne, Dole, 1619, p. 299 sqq. 
qu'un demi niquet par charge " de sel (soit environ $100 \mathrm{~kg}$ ). Autrement dit, elle est payé à la pièce ${ }^{98}$. Surtout, Bonne Delezet ajoute un renseignement précieux concernant le travail de nuit, dont la fatigue accumulée a des répercussions sur le travail à l'aube : " Ayant travaillé toute la nuict, quelque fois sur le matin l'on se trouve endormi, qui est cause que l'on tourne la main quelque fois plus qu'il ne convient ${ }^{99}$."

Les souverains ont toujours versé des pensions à leurs bons serviteurs ${ }^{100}$. Les ducs puis les comtes de Bourgogne protègent leurs ouvriers fidèles, lorsqu'ils parviennent au terme de leur vie. L'aide sociale apportée aux " povres ouvrieres et ouvriers " est souhaitée par les officiers de la saline dans une requête adressée dès 1443 au duc de Bourgogne ${ }^{101}$. La nature de cette aide nous échappe. En revanche, nous retrouvons nos femmes "privilégiées " sollicitant des pensions ${ }^{102}$. Anne, femme de Jehan Maillet, adresse ainsi une requête au conseil de la saunerie en 1476, disant qu'elle y travaille "depuis et sont environ 38 ans ", ayant servi avec diligence, pour "seschier les selz en ladite barne de Mertenet ". Sa vie n'a pas été facile: " pendant lequel temps icelle a eu charge de plusieurs gessines d'enffans dont elle est présentement bien soulée et abattue "! Trop âgée, Anne ne peut plus exercer son métier. Le conseil doit donc donner un avis pour accorder à cette ouvrière une " pencion par sepmaine pour la aidier à vivre comme il est de bonne coustume faire et donner en ladite saulnerie aux ouvriers et ouvrieres d'icelle, quant ilz ont bien et longuement servie sans répréhension quelconque ". Comme Anne a donné satisfaction, le conseil lui offre une pension hebdomadaire; elle recevra " chacun dymanche son salère ${ }^{103}$ comme les autres ouvriers et ouvrières de cestedite saulnerie et pour une fois deux bénastes de sel bouché marqué ${ }^{104}$ ".

En 1619, Alix Marguet est « estignarix, en l'exercice de laquelle elle a fait tant de debvoir et travaille si diligement et fidellement que maintenant elle s'en treuve fort caducque et commodé de sa personne et tel-

98. Nous laissons de côté la question du salaire des femmes, qui nécessitera une approche détaillée tenant compte des avantages en nature (sel, logement, etc.).

99. Arch. dép. du Doubs, 2 B 3341.

100. Delsalle, Paul, La France industrielle..., op. cit., p. 148-150.

101. Arch. dép. du Doubs, B 238.

102. Arch. dép. du Doubs, B 187, $\mathrm{f}^{\circ} 89 \mathrm{v}^{\circ}$; " Des ouvrières ayant servi 50 ans ou environ obtiennent une pension de 3 sols par semaine, plus un "salé", provision de sel pour la semaine. Certains bénéficiaires n'obtiennent que le "salé". On donne un panier de sel à toute ouvrière qui "git d'enffant" ": DuBoIs, Henri, article cité, Wirtschaftskräfte und Wirtschaftswege, (Festschrift für Hermann Kellebenz), p. 204. Sur l'origine de l'aide sociale aux salines de Salins, cf. BROCARD, Nicole, Soins, secours et exclusion. Établissements hospitaliers et assistance dans le diocèse de Besançon, XIV et XV $V^{e}$ siècles, Besançon, Pufc, 1998, p. 210.

103. On notera au passage l'utilisation du mot salaire, ici sous la forme salère, directement dérivé du mot sel (salarium : ration de sel).

104. Arch. dép. du Doubs, B 187, f 189 v ; FAUDOT, Nathalie, Recherches sur la grande saunerie de Salins (1476-1477), Université de Franche-Comté, mémoire de maîtrise, 1988, p. 69; la transcription donnée en fin de volume indique le mot " gessmes " qui ne signifie rien; c'est pourquoi je propose "gessines". 
lement foible qu'elle ne peult plus supporter seulle le grand travail que requiert tel œuvre ». Elle demande « l'aulmosne ou recompense ordinaire ». Le conseil des finances accorde "sa vie durant " 8 gros par semaine ${ }^{105}$. Clauda Vauchet expose elle-même son cas, parfaitement comparable, dans une requête adressée en 1642 au comte de Bourgogne, le roi Philippe IV. Elle demande une petite pension pour vivre, sa situation étant difficile au soir de sa vie:

"Au roy tres catholique,

Expose en toute humilité Clauda Vauchet metatrix en l'ouvroir de la berne de Glappin, en votre grande saulnerie de Salins, que sont passéz quarante [souligné] ans, elle est entremise au service de votre majesté, tant audit estat qu'aultres, qu'elle a exercé ausdites saulneries avec telle vigilence et sincerité qu'elle n'a donné sujet aucun de mescontentement a qui que ce soit, mesmement durant toutes les pestes qui ont regné audit Salins l'an 1629 , auquel temps encor que tous les aultres officiers eussent quitté leurs besoignes, et qu'a peine se trouva il personne qui voulu servir ausdites saulneries, a cause de la violence du mal; elle toutefois ne desista aucunement ains persista jusqu'à y estre frappée mesme de peste, et perdre tout le peu qu'elle avoit, tant a payer les fraiz du nettoyement, qu'aultres, en suitte ce qui l'a tellement espuisée de commodités temporelles, et encor de forces corporelles, avec l'aage de plus de soixante ans [souligné], qu'elle se void incappable d'exercer sa charge, si ce n'est par aultruy et a ses frais ${ }^{106}$; lesquels frais ne pouvant supporter a cause de l'extreme cherté, causée par les guerres, ny mesme vivre de soy, si ce n'est qu'il plaise a V. Magesté luy vouloir accorder au moins la mesme recompence qu'elle a coustume de conceder aux ouvriers et ouvrieres qui ont rendu quelque service (voire moindre qu'elle) ausdites saulneries, qui est deux gros en argent, et deux gros salés [= pains de sel], par chasque sepmaine, sa vie naturelle durant. C'est de quoy elle supplie tres humblement V.M., et ce faisant elle priera pour sa santé, prospérité et salut ${ }^{107}$."

Bien lui en a pris car le Conseil des finances du roi, à Bruxelles, lui accorde six gros par semaine. Toutes les vieilles femmes ne demandent pas une pension de retraite, même lorsque leur âge est très avancé. En 1630,

105. Archives générales du royaume de Belgique, Conseil des finances, ${ }^{\circ} 69$; décision prise à Mariemont, résidence des Archiducs, le 12 juin 1619. On notera que les sommes accordées diffèrent selon les ouvrières.

106. Le système du remplacement est dénoncé dans une ordonnance du 26 janvier 1565 : «Et pour ce que les officiers de ladite saunerie aians autorité de y commettre les ouvrières et ouvriers pourvoient esdites charges bien souvent leurs serviteurs servantes ou nourrices lesquels, pour encore estre retenues aux services de leurs maistres et maîtresses font desservir leursdits charges et manœuvres par quelques pauvres gens prenans d'eux un pot de vin ou portion de leurs petits gaiges, que peut causer qu'ils n'en versent fidellement en leurdites charges, saite Altesse veut et ordonne que tous ceux qui auront charge de manœuvre en ladite saunerie seront tenus de desservir en personnes sans pouvoir commettre d'autres en leurs lieux à peine d'en estre privez "; Arch. dép. du Jura, 1 F 183.

107. Archives générales du royaume de Belgique, Conseil des finances, $n^{\circ} 69$; la requête n'est pas autographe. En marge de la feuille déchirée, on peut lire : "Claude Nicolot pbre de lorat [prêtre de l'Oratoire?] de Salins "; s'agit-il de l'intermédiaire? 
Clauda Amadry, veuve de Jacques Parrel, travaille encore dans la saline, en compagnie de sa fille, en tant que fassary depuis 60 ans : elle est âgée de 80 ans! Or, elle apparaît simplement comme témoin, déposant dans une affaire en justice ${ }^{108}$.

La seconde moitié du XVIII ${ }^{\mathrm{e}}$ siècle est, pour Salins devenue française, une période de grande mutation : l'élévation du prix due à la raréfaction du bois entraîne la décision de construire une nouvelle saline à côté de la forêt de Chaux, en y dérivant la saumure. L'architecte Claude-Nicolas Ledoux est chargé d'édifier le complexe industriel entre les villages d'Arc et de Senans. Le nombre d'ouvrières semble alors beaucoup plus faible qu'aux siècles antérieurs. Un "état des ouvriers et ouvrières " ne dénombre que cent dixsept ouvriers et ouvrières, à la fin du siècle ${ }^{109}$. Cette éviction progressive de la main-d'œuvre féminine se produit en même temps dans les salines bavaroises : les femmes sont remplacées par des hommes au cours de la réforme mise en œuvre par Johann Sebastian Clais; comme les enfants, elles ne peuvent même plus entrer dans la saline, sauf pour apporter les repas aux hommes ${ }^{110}$. Les rares auteurs ayant évoqué la main-d'œuvre de Salins et de Chaux n'attribuent aux femmes que des "travaux subalternes ", un peu comme à Bad Reichenhall, ce qui ne correspond pas à la réalité, même au XVIII ${ }^{\mathrm{e}}$ siècle $^{111}$. Certaines femmes continuent à dominer la confection des pains de sel ${ }^{112}$. Des fassaris et des mettaris sont toujours présentes ; certaines, démunies, n'hésitent pas à voler des pains de sel en les dissimulant sous leurs jupes ${ }^{113}$. Il y a donc une certaine pérennité du système même si des changements dans l'entreprise ont contribué à la progressive éviction de quelques femmes et à leur remplacement par des hommes.

En définitive, la mise en ouvre du sel, à Salins, est pour une large part entre des mains féminines. Serait-il exagéré de penser que la confection des pains de sel s'apparente à des opérations culinaires, confiées plus aisément aux femmes ${ }^{114}$ ? Il est difficile de cerner leur statut exact, leur place sociale aussi. Elles ont un poste de responsabilité même si elles encadrent très peu

108. Arch. dép. du Doubs, 2 B 3341.

109. Arch. dép. du Jura, A 90; A 106; A 107.

110. StA München, Salzmaieramt Reichenhall B 5.; « Von " nassen und truckenen Dirnen ». Frauenarbeit in den bayerischen Salinen ", Salz macht Geschichte. Aufsätze, op. cit., p. 142-147.

111. $C f$. "Von " nassen und truckenen Dirnen ». Frauenarbeit in den bayerischen Salinen ", Salz macht Geschichte. Aufsätze..., op. cit., p. 144; à Reichenhall, elles ne supervisent pas la « cuisine " du sel; elles alimentent le feu en bois, enlèvent les cendres.

112. GALLET, Michel, Claude-Nicolas Ledoux, 1736-1806, Paris, Picard, 1980, p. 106-107.

113. Par exemple, Denise Quantin, mettari, qui a 19 ans en 1738, dissimule deux pains de sel sous ses jupes; les gardiens qui fouillent le personnel découvrent parfois une poche cousue sous la jupe. Cf. Vernus, Michel, " Faux sauniers et faux saunage dans le Jura (XVIII siècle)", Actes du $107^{e}$ congrès national des sociétés savantes (Brest, 1982), Paris, CTHS, Histoire moderne et contemporaine, tome 1, p. 207-225.

114. De même, sur les chantiers du bâtiment et des fortifications, nous constatons aussi que la confection de la chaux est souvent confiée aux femmes; s'agirait-il d'une opération assimilable à de la cuisine? Cf. DELSALLE, Paul, "Le travail des femmes sur les chantiers et dans les métiers du bâtiment, aux Xv" $\mathrm{XVI}^{\mathrm{e}}$ et XVII ${ }^{\mathrm{e}}$ siècles ", L'Edilizia prima della 
de main-d'œuvre, exception faite des « gardes ». Derrière le mot " ouvrière " ou " manœuvre " se cache parfois une véritable technicienne spécialisée, voire une contremaîtresse. Leur place, stratégique, se situe bien au cœur de la production des salines. Elles dominent techniquement la confection des pains de sel; ce n'est pas rien. Il est surprenant que le cas des ouvrières de Salins n'ait pas attiré plus tôt l'attention des historiens du monde ouvrier. N'est-il pas étonnant que les principaux ouvrages d'histoire économique et sociale aient négligé l'approche de la main-d'œuvre, notamment féminine, ici et ailleurs?

Jusqu'à la veille de 1789 , le sort des femmes ouvrières dans cette saline de Salins est un des plus surprenants qu'on puisse imaginer, très loin de ce qui a été perçu et écrit. La situation est très différente de celle observée dans d'autres salines d'Europe, par exemple à Hallein, à Halle ou encore à Reichenhall en Baviève : là, elles sont minoritaires et surtout subalternes des hommes ${ }^{115}$. Au contraire, à Salins, les femmes sont présentes en grand nombre dans la saline et, de surcroît, certaines d'entre elles occupent des postes de responsabilité au sein de la grande usine. On y observe aussi la quasi-hérédité de certaines charges ou offices, transmissibles de mère en fille, et même l'existence d'un système de versement de pensions de retraite. C'est peu de chose, certainement, mais les entreprises offrant cela, surtout aux femmes, sont alors très rares. Enfin, rien n'est sans doute plus singulier que ces destins féminins longtemps restés anonymes, ces dizaines d'années passées à travailler dans la saunerie, autour des cuves d'évaporation et de cuisson, jour et nuit. Comment ces ouvrières ont-elles pu survivre et devenir sexagénaires, septuagénaires ou octogénaires ${ }^{116}$ ?

Rivoluzione industriale, secc. XIII-XVIII, Prato, Istituto Internazionale di Storia Economica F. Datini, Firenze, Le Monnier, 2005, p. 861-887.

115. WEBER, Renate, "Von " nassen und truckenen Dirnen ». Frauenarbeit in den bayerischen Salinen ", Salz macht Geschichte. Aufsätze, op. cit., p. 142-147; HocQUET, JeanClaude, Le sel..., op. cit., p. 52; PІесноскI, W., Die Halloren, Geschichte und Tradition der "Salzwirkerbrüderschaft im Thale zu Halle », Leipzig, 1981.

116. On peut rester sceptique devant cette longévité des ouvrières. Pourtant d'autres témoignages locaux de la même époque confirment une espérance de vie qui, parfois, est longue (jusqu'à 110 ans et 112 ans). L'étude des registres paroissiaux, en cours, devrait corroborer ces déclarations. 


\section{RÉSUMÉ}

À la fin du Moyen Âge et aux siècles suivants, l'activité féminine est notoire surtout dans l'industrie textile. On a moins prêté attention à d'autres secteurs industriels, notamment celui des salines, où l'on trouve des femmes, parfois en grand nombre. Il ne s'agit aucunement de paysannes qui, de façon temporaire, pourraient louer leurs bras mais de véritables salariées à plein temps. Les archives exceptionnelles des salines de Salins, dans le massif du Jura, permettent l'étude des ouvrières jusqu'à la fin du XVII' ${ }^{e}$ siècle. Elles soulèvent surtout la question de la définition et du statut de "l'ouvrière " ou de la " manouvre ". Derrière ces termes assez vagues se cache une grande variété d'emplois féminins, mais surtout des spécialisations et des responsabilités parfois très surprenantes. Le sort de la femme ouvrière dans cette saline de Salins est un des plus singuliers qu'on puisse imaginer. La situation est très différente de celle observée dans d'autres salines d'Europe. A Salins, les femmes sont présentes en grand nombre dans la saline et, de surcroît, certaines d'entre elles occupent des postes de responsabilité au sein de la grande usine, pendant plusieurs dizaines d'années consécutives. On y observe la quasihérédité de certaines charges ou offices, et même l'existence d'un système de versement de pensions. C'est peu de chose, certainement, mais les entreprises offrant cela, surtout aux femmes, sont alors très rares.

\section{ABSTRACT}

At the end of the Middle Age and during the following centuries, feminine activity especially in the textile industry is well known. Less attention was paid to other industrial sectors, saltworks among others, where sometimes numbers of women also worked. They, in no way were peasant women whose services would, on a temporary basis, have been hired, but real full time salaried employees. The rousing archives of the saltworks in Salins, in the Mountains of Jura, enable to the study of the female wage earners up to the end of the $18^{\text {th }}$ century. That raises the issue of the "wage earner" or "unskilled worker's" definition and status. These quite vague terms hire a wide range of feminine jobs, and especially sometime very surprising specializations and responsibilities. In this saltwoks in Salins, the female wage earner lot is one of the most peculiar that one can imagine. The situation is very different compared to other well known European saltworks. In Salins, women were quite numerous and, moreover, some of them hold during several ten consecutive years a position of responsibility inside the big plant. One can notice the almost hereditary nature of some task or offices and, also the existence of a pension payment system. It certainly was not much, but at that time just a few number of corporations would offer such conditions to female workers. 